\title{
Childhood Obesity - An Emerging Problem : A Review Article
}

\author{
FARZANA HAMID ${ }^{1}$, RAFIQUL ISLAM ${ }^{2}$, PROKASH CHANDRA RAY ${ }^{3}$
}

Introduction:

Childhood obesity is now among the most widespread medical problems affecting children and adolescents living in both developed \& developing countries. Obesity is among the easiest medical conditions to recognize but most difficult to treat. Unhealthy weight gain due to improper diet and lack of exercise is responsible for over 300,000 deaths each year worldwide. ${ }^{1}$ Overweight children are much more likely to become overweight adults unless they adopt and maintain healthier patterns of eating and exercise. Approximately $21-24 \%$ of children and adolescents are overweight, and another $16-18 \%$ is obese. ${ }^{2}$ The prevalence of obesity is increasing in all paediatric age groups, in both sexes, and in various ethnic and racial groups. Many factors, including genetics, environment, lifestyle, and eating habits are believed to play a role in the development of obesity. However, more than $90 \%$ of cases are idiopathic; less than $10 \%$ are associated with hormonal or genetic causes. ${ }^{3}$ Obesity has a profound effect on a patient's life. Childhood obesity predisposes to insulin resistance and type 2 diabetes, hypertension, hyperlipidemia, liver and renal disease, and reproductive dysfunction. This condition also increases the risk of adult-onset obesity, cardiovascular disease \& can create emotional and social problems. Therefore, the prevention and treatment of obesity have emerged as a challenge to the paediatric practitioner. ${ }^{3}$

\section{Epidemiology:}

The epidemiology of obesity during childhood varies by age, gender, race, ethnicity and other factors. ${ }^{4}$ However, Eneli and Dele Davies reported that in $77 \%$ countries, the prevalence rate of childhood obesity is $10 \% .{ }^{5}$ Notably, the highest rates for children at risk for obesity were found in Malta (25.4\%) and the United States (25.1\%). Lithuania (5.1\%) and Latvia (5.9\%) had the lowest rates. ${ }^{5}$ A recent European Youth Heart

1. Assistant Professor, Dept. of Paediatrics, Delta Medical College, Dhaka

2. Professor, Dept. of Paediatrics, Delta Medical College, Dhaka

3. Resident Physician, Dhaka Mohanagar Sishu Hospital

Correspondence: Dr.Farzana Hamid MRCPCH (UK), FCPS

(Paed), Tel: 01815474442
Study suggests, Swedish children have a lower risk of becoming overweight or obese in adolescence compared with Estonian children. ${ }^{4}$ Adolescent obesity is predictive of adult obesity, with $80 \%$ of teenagers who are obese continuing to be obese as adults. Obesity is more likely to occur during specific periods of life, such as when children are aged 5-7 years and during adolescence. A recent European Youth Heart Study suggests male sex confers a higher risk of obesity in adolescence. ${ }^{4}$

\section{Definition:}

Obesity means an excess amount of body fat. No general agreement exists on the definition of obesity in children and adolescents, unlike standards for adults. Nevertheless, most professionals accept published guidelines based on the body mass index (BMI) modified for age, pubertal stage, and gender to measure obesity in children and adolescents. ${ }^{3}$ Others define pediatric obesity as body weight at least $20 \%$ higher than the healthy weight range for a child or adolescent of that height, or as a body fat percentage above $25 \%$ in boys or above $32 \%$ in girls. ${ }^{2}$

\section{Body Mass Index (BMI):}

The BMI is a continuous measure of body fatness. It is calculated as weight in kilograms $(\mathrm{kg})$ divided by height $\left(\mathrm{m}^{2}\right)$. The BMI correlates closely with total body fat (TBF), which is estimated using dual-energy $x$-ray absorptiometry (DEXA) scanning in children who are overweight and obese. ${ }^{6}$ Normal values for BMI vary with age, sex, and pubertal status, and standard curves representing the 5th through the 95th percentiles for $\mathrm{BMI}$ in childhood and adolescence . .,6 Consensus committees have recommended that children and adolescents are considered overweight or obese if the BMl exceeds the 85th or 95th percentiles or exceeds $30 \mathrm{~kg} / \mathrm{m}^{2}$ at any age. ${ }^{3}$

Table-I

Body Mass Index (BMI) classification of children \& adolescent $^{3}$

\begin{tabular}{ll}
\hline BMI percentile for age & Weight status \\
$<5^{\text {th }}$ percentile & Underweight \\
$5^{\text {th }}-84^{\text {th }}$ percentile & Normal weight \\
$85^{\text {th }}-94^{\text {th }}$ percentile & Overweight \\
$>95^{\text {th }}$ percentile & Obese \\
\hline
\end{tabular}




\section{Etiology and Pathophysiology:}

The etiologies of obesity are complex and include genetic, biological, behavioral and cultural factors. ${ }^{1-7}$

\section{- Genetic factors}

- Obesity tends to run in families.

- A child with an obese parent, brother, or sister is more likely to become obese.

\section{- Dietary habits}

- The dietary habits of children and teenagers have shifted away from healthy foods (such as fruits, vegetables, and whole grains) to a much greater reliance on fast food, processed snack foods, and sugary drinks.

- These foods tend to be high in fat and/or calories and low in many other nutrients.

- Several patterns are associated with obesity. Unhealthy habits include eating when not hungry, eating while watching TV or doing homework, or drinking sodas during sedentary activities (like at the movies or watching TV).

\section{- Physical inactivity}

- The popularity of television, computers, and video games has translated into an increasingly sedentary lifestyle for many children and teenagers. They, on average, spend over three hours daily watching television. This form of recreation uses little energy (calories).

- Only one-third of children have daily physical education at school.

- Parents' busy schedules and even fears about public safety prevent many children and teenagers from taking part in sports, or other activity programs after school.

- Genetic syndromes

- Prader-Willi syndrome

- Pseudohypoparathyroidism

- Laurence-Moon-Biedl syndrome

- Down syndrome

- Turner syndrome

- Hormonal disorders

- Growth hormone deficiency

- Hypothyroidism

- Glucocorticoid excess (Cushing syndrome)

- Precocious puberty

- Polycystic ovary syndrome (PCOS)

\section{- Medications}

- Cortisol and other glucocorticoids

- Sulfonylureas

- Tricyclic antidepressants (TCAs)

- Monoamine oxidase inhibitors (MAOIs), such as phenelzine

- Oral contraceptives

- Insulin (in excessive doses)

- Risperidone

- Clozapine

Obesity results from a dysregulation of calorie intake and energy expenditure. ${ }^{2}$ Dysfunction in the gut-brainhypothalamic axis via the ghrelin/leptin hormonal pathway has been suggested to have a role in abnormal appetite control and excess energy intake. ${ }^{3}$ Studies indicate that dysfunction in this hormonal axis may be the causative factor in as many as $10 \%$ of obese subjects. One study reported an increased incidence of obesity at age 3 years in infants weaned to solid foods by 4 months. ${ }^{6}$ The accumulation of body fat, particularly in a visceral distribution, reduces the sensitivity to insulin in skeletal muscle, liver tissue, and adipose tissue; this "insulin resistance" predisposes to glucose intolerance and hypertriglyceridemia. ${ }^{7}$ Low levels of high-density lipoprotein (HDL), observed both genetically and in association with a sedentary lifestyle, likely contribute to the increase of premature coronary artery disease in obesity. The insulin resistance, dyslipidemia, and hypertension predispose to type 2 diabetes and cardiovascular disease, reducing life expectancy. ${ }^{8}$

\section{Evaluation of obese children:}

Evaluation of obese children requires sensitivity and compassion as they often have decreased self esteem. The initial evaluation is focused on exploring dietary practices, family structure, and habits because alteration of these factors is usually the basis of successful treatment. It is also important to determine if there may be an underlying secondary cause of obesity or if there are current comorbidities from being overweight. 3,7

Short stature or a reduced rate of linear growth in a child with obesity suggests the possibility of growth hormone deficiency, hypothyroidism, cortisol excess, pseudohypoparathyroidism, or a genetic syndrome such as Prader-Willi syndrome. ${ }^{6}$ 
A history of dry skin, constipation, intolerance to cold, or fatigability suggests hypothyroidism.

Polyuria and polydipsia may be noted if the adolescent with obesity develops overt diabetes. Acanthosis nigricans suggests insulin resistence.

A history of damage to the central nervous system (CNS) (eg, infection, trauma, hemorrhage, radiation therapy, seizures) suggests hypothalamic obesity with or without pituitary growth hormone deficiency or pituitary hypothyroidism. A history of morning headaches, vomiting, visual disturbances, and excessive urination or drinking also suggests that the obesity may be caused by a tumor or mass in the hypothalamus. ${ }^{8}$

Selective accumulation of fat in the neck, trunk, and purple striae suggest an excess of cortisol, particularly if the rate of linear growth has declined. Measurement of blood pressure is very important.

The appearance of signs of sexual development at an early age suggests that the weight gain is caused by precocious puberty. However, excessive facial hair, acne, and irregular periods in a teenage girl suggest that the weight gain may be caused by cortisol excess or polycystic ovary syndrome (PCOS). Obesity itself may be accompanied by facial hair, irregular menses, and hypertension. ${ }^{3,6-8}$

\section{Differential Diagnosis:}

- Growth Hormone Deficiency

- Hypothyroidism

- Cushing Syndrome

- Diabetes

- Polycystic Ovarian Disease

- Prader-Willi Syndrome

- Precocious Puberty

\section{Work up:}

The following laboratory studies may be indicated in patients with obesity 3,8

- Fasting and 2-hours postprandial blood glucose

- Blood insulin level

- Fasting lipid panel for detection of dyslipidemia

- Thyroid function tests

- Adrenal function tests, when indicated, to assess the possibility of Cushing syndrome

- Growth hormone secretion and function tests, when indicated
- Serum calcium, phosphorus, and parathyroid hormone levels to evaluate for suspected pseudohypoparathyroidism

- Karyotype (with fluorescence in situ hybridization [FISH] for Prader-Willi [15q-]), when indicated by clinical history and physical examination

- Assessment of reproductive hormones (including prolactin), when indicated

- Magnetic resonance imaging (MRI) of the brain with focus on the hypothalamus and pituitary , when indicated

\section{Management:}

Successful treatment of obesity is challenging, and treatment goals vary, depending on the age of the child and the severity of complications. ${ }^{3}$ Children are still growing, so severe caloric restriction and weight loss may be detrimental. An initial goal of $10 \%$ reduction in weight is reasonable. Once achieved, the new weight should be maintained for 6 months before further weight loss is attempted. ${ }^{11}$

Obesity is best managed by a multidisciplinary team. ${ }^{10}$ Teams may include paediatrician, endocrinologist, dietitian, exercise specialist, psychologist, nurse, and counselor. Management consists of $3,10,11$

\section{Dietary counseling}

- Exposure to a wide variety of foods, including less calorie dense food choices and limitation of between-meal snacking

- Encourage children to eat slowly. This helps them recognize the feeling of fullness and stop eating when they are full.

- Sweetened beverages should be limited and parents should continue to offer healthy foods

- Foods should not be used as reward

- Foods should be offered at multiple times. Repeated exposure to initially disliked foods will break down resistance

- Children tend to be more aware of satiety, so allow children to respond to satiety. Parents should not force children to clean their plate.

Lifestyle Modifications, Exercise and Physical Activity:

- Decreased sedentary activity

- Avoiding television \& computer in children younger than 2 years of age 
- Children 2-18 years of age should have $<2 \mathrm{hr} /$ day of screen time

- Encourage children and teenagers to enjoy physical activities that burn calories and use different muscle groups. These include games which involve walking, running, swimming or riding a bicycle.

- Encourage children to get involved in sports at school or in the community

- Graded exercise regimen is useful

\section{Medications:}

Pharmacologic treatment in children is reserved for those with severe medical complications. Regarding the use of anorectic agents, a 12-month, randomized placebo-controlled trial of sibutramine in 498 adolescents demonstrated a significant, drugassociated reduction in body mass index (BMI) (sibutramine, $8.2 \%$ vs placebo, $0.8 \%$ ) without any observed cardiodynamic effects. ${ }^{12}$ Another multicenter, randomized trial of orlistat in obese adolescents demonstrated weight stabilization and reduced body fat in the orlistat group, whereas significant weight gain was observed in patients receiving placebo. ${ }^{13} \mathrm{Administration}$ of anorectic drugs may be considered in the postpubertal adolescent, but only after the patient has failed to respond to vigorous attempts to modify behavior, diet, and family interactions. ${ }^{13}$

\section{Surgery:}

The American Paediatric Surgical Association Guidelines recommend that surgery be considered only in children with a BMI > 40 and a medical complication of obesity after they have failed 6 months of a multidisciplinary weight management program. ${ }^{14}$

\section{Long-Term Monitoring:}

Regular follow-up is indicated in patients with obesity for the following reasons ${ }^{15}$

- Reinforcement of nutritional goals and exercise objectives

- Identification of social and emotional barriers to therapy

- Family support and counseling (if indicated)

- Assessment of growth, pubertal development, and reproductive function

- Assessment of glucose tolerance and fasting lipid levels
- Identification and management of obesity-related acute and chronic complications

\section{Complications:}

There are many risks and complications with obesity 3,16

- Insulin resistance, Type 2 Diabetes Mellitus

- Hypertension

- Dyslipidemia

- Pulmonary complication (Increased frequency of reactive airways, poor exercise tolerance, increased work of breathing, and increased oxygen consumption)

- Musculoskeletal problems (Slipped capital femoral epiphysis, Blount disease)

- Cholelithiasis

- Fatty liver disease

- Obstructive sleep apnea

- Polycystic ovary syndrome

- Pseudotumor cerebri

- Emotional and psychosocial problems (eg, suicide attempts, manic depression, other depressive disorders)

- Gout and colorectal cancer increased among men who had obesity as adolescents, and arthritis increased among women who had obesity as adolescents.

\section{Prevention:}

Preventing the development of obesity in childhood and adolescence is more likely to reduce long-term complications in adults. In 2008, The Endocrine Society released guidelines for the prevention and treatment of childhood obesity. Proposed suggestions for the prevention of obesity are as follows ${ }^{3,15,17}$

\section{Pregnancy:}

- Normalize BMI before pregnancy

- Maintain moderate exercise as tolerated

- Avoid smoking

- In gestational diabetes, provide meticulous glucose control

Postpartum \& Infancy:

- Breast feeding for 6 months

- Postpone introduction of solid food \& sweet liquid 


\section{Families:}

- Eat meals as a family in a fixed place \& time

- Do not skip meals, especially breakfast

- No television during meals

- Avoid unnecessary sweet or fatty foods \& soft drinks

\section{Schools:}

- Educate children from primary school on appropriate diet \& life style

- Mandate physical education, including $30 \mathrm{~min}$ of exercise 2-3 times weekly

- Encourage the walking school bus

\section{Communities:}

- Increase family friendly exercise \& play facilities for children of all age

- Discourage use of elevators \& moving walkways

\section{References:}

1. Dietz WH, Robinson TN. Overweight children and adolescents. N Engl J Med 2005; 352: 2100-2109.

2. Gagagan S. Child and adolescent obesity. Curr Probl Pediatr Adolesc Health Care 2004; 34: 1-48.

3. Gahagan S. Overweight and obesity. In: Behrman RE, Kliegman RD, Jenson HB editors Nelson Text book of Paediatrics. Philadelphia: WB Saunders 2004; P. 179-88.

4. Ogden CL, Yanovski SZ, Carroll MD, Flegal KM. The epidemiology of obesity. Gastroenterology 2007; 132:2087-2102.

5. Eneli I, Dele Davis H. Epidemiology of childhood obesity. In: Dele Davis $\mathrm{H}$, ed. Obesity in Childhood \& Adolescence. Westport, Conn: Praeger Perspectives. 2008: 3-19.

6. Ogden CL, Carroll MD, Kit BK, Flegal KM. Prevalence of obesity and trends in body mass index among US children and adolescents. JAMA 2012; 307(5): 483-90.

7. QuattrinT, Liu E, Shaw N. Obese children who are referred to the pediatric endocrinologist: Characteristics and outcome. Pediatrics 2005; 115: 348-51.
8. Maffeis C, Pinelli L, Brambilla P, Banzato C, Valzolgher L, Ulmi D, et al. Fasting plasma glucose and the risk of impaired glucose tolerance in obese children and adolescents. Obesity 2010; 18: 1437-42.

9. Oude Luttikhuis $\mathrm{H}$, Baur L, Jansen $\mathrm{H}$. Interventions for treating obesity in children. Cochrane Database Syst Rev 2009; CD001872.

10. Kalarchian MA, Levine MD, Arslanian SA. Family-based treatment of severe pediatric obesity: randomized, controlled trial. Pediatrics 2009; 124: 1060-8.

11. Jolly K, Lewis A, Beach J, Denley J, Adab P, Deeks JJ, et al. Comparison of range of commercial or primary care led weight reduction programmes with minimal intervention control for weight loss in obesity: Lighten Up randomised controlled trial. BMJ 2011; 343: d6500.

12. August GP, Caprio S, Fennoy I. Prevention and treatment of pediatric obesity: an endocrine society clinical practice guideline based on expert opinion. J Clin Endocrinol Metab 2008; 93: 4576-99.

13. Dunican KC, Desilets AR, Montalbano JK. Pharmacotherapeutic options for overweight adolescents. Ann Pharmacother. 2007; 41: 1445-55.

14. Elder KA, Wolfe BM. Bariatric surgery: A review of procedures and outcomes. Gastroenterology 2007; 132: 2253-71.

15. Marcus C, Nyberg G, Nordenfelt A, Karpmyr M, Kowalski J, Ekelund U,et al. A 4-year, clusterrandomized, controlled childhood obesity prevention study: STOPP. Int J Obes 2009; 33 :408-17.

16. McGavock JM, Torrance BD, McGuire KA, Wozny PD, Lewanczuk RZ. Cardiorespiratory fitness and the risk of overweight in youth: the Healthy Hearts Longitudinal Study of Cardiometabolic Health. Obesity 2009; 17 : 1802-7.

17. Waters E, de Silva-Sanigorski A, Hall BJ. Interventions for preventing obesity in children. Cochrane Database Syst Rev 2011; 12: CD001871. 